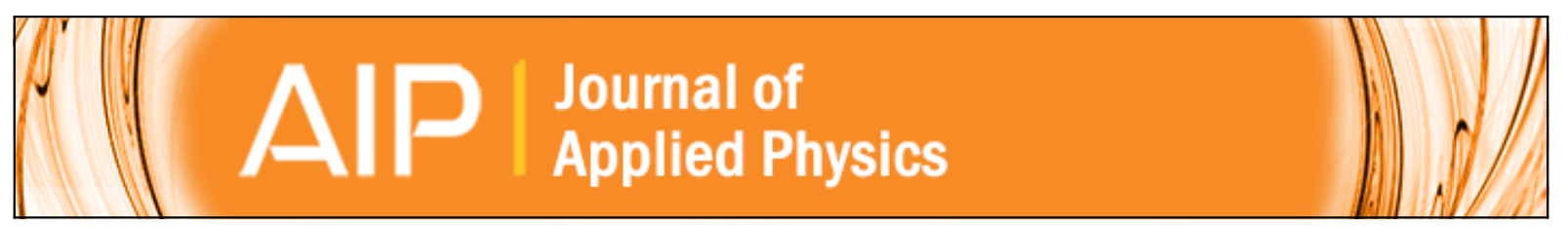

\title{
Double-gated Si NW FET sensors: Low-frequency noise and photoelectric properties
}

F. Gasparyan, H. Khondkaryan, A. Arakelyan, I. Zadorozhnyi, S. Pud, and S. Vitusevich

Citation: Journal of Applied Physics 120, 064902 (2016); doi: 10.1063/1.4960704

View online: http://dx.doi.org/10.1063/1.4960704

View Table of Contents: http://scitation.aip.org/content/aip/journal/jap/120/6?ver=pdfcov

Published by the AIP Publishing

\section{Articles you may be interested in}

Single trap dynamics in electrolyte-gated Si-nanowire field effect transistors

J. Appl. Phys. 115, 233705 (2014); 10.1063/1.4883757

Laser direct writing of silicon field effect transistor sensors

Appl. Phys. Lett. 102, 093504 (2013); 10.1063/1.4794147

A pH sensor with a double-gate silicon nanowire field-effect transistor Appl. Phys. Lett. 102, 083701 (2013); 10.1063/1.4793655

Theory of signal and noise in double-gated nanoscale electronic $\mathrm{pH}$ sensors

J. Appl. Phys. 112, 034516 (2012); 10.1063/1.4737604

Optimal signal-to-noise ratio for silicon nanowire biochemical sensors

Appl. Phys. Lett. 98, 264107 (2011); 10.1063/1.3608155

\section{Pure Metals • Ceramics} Alloys $•$ Polymers in dozens of forms 


\title{
Double-gated Si NW FET sensors: Low-frequency noise and photoelectric properties
}

\author{
F. Gasparyan, ${ }^{1,2}$ H. Khondkaryan, ${ }^{1}$ A. Arakelyan, ${ }^{1}$ I. Zadorozhnyi, ${ }^{2}$ S. Pud, ${ }^{2}$ \\ and S. Vitusevich ${ }^{2, a)}$ \\ ${ }^{1}$ Yerevan State University, 1 Alex Manoogian St., 0025 Yerevan, Armenia \\ ${ }^{2}$ Forschungszentrum Jülich, Peter Grünberg Institute (PGI-8), 52425 Jülich, Germany
}

(Received 4 May 2016; accepted 29 July 2016; published online 10 August 2016)

\begin{abstract}
The transport, noise, and photosensitivity properties of an array of silicon nanowire (NW) $\mathrm{p}^{+}-\mathrm{p}-\mathrm{p}^{+}$ field-effect transistors (FETs) are investigated. The peculiarities of photosensitivity and detectivity are analyzed over a wide spectrum range. The absorbance of p-Si NW shifts to the short wavelength region compared with bulk $\mathrm{Si}$. The photocurrent and photosensitivity reach increased values in the UV range of the spectrum at $300 \mathrm{~K}$. It is shown that sensitivity values can be tuned by the drain-source voltage and may reach record values of up to $2-4 \mathrm{~A} / \mathrm{W}$ at a wavelength of $300 \mathrm{~nm}$ at room temperature. Low-frequency noise studies allow calculating the photodetectivity values, which increase with decreasing wavelength down to $300 \mathrm{~nm}$. We show that the drain current of $\mathrm{Si}$ NW biochemical sensors substantially depends on $\mathrm{pH}$ value and the signal-to-noise ratio reaches the high value of $10^{5}$. Increasing $\mathrm{pH}$ sensitivity with gate voltage is revealed for certain sourcedrain currents of $\mathrm{pH}$-sensors based on Si NW FETs. The noise characteristic index decreases from 1.1 to 0.7 with the growth of the liquid gate voltage. Noise behavior is successfully explained in the framework of the correlated number-mobility unified fluctuation model. $\mathrm{pH}$ sensitivity increases as a result of the increase in liquid gate voltage, thus giving the opportunity to measure very low proton concentrations in the electrolyte medium at certain values of the liquid gate voltage. Published by AIP Publishing. [http://dx.doi.org/10.1063/1.4960704]
\end{abstract}

\section{INTRODUCTION}

Over the past decade, silicon nanowires (NWs) have been under intensive study due to their promising electrical, optical, chemical, thermal, and mechanical properties. Because of their large surface-to-volume ratio, easy functionalization, and modification, devices based on Si NWs are of decisive importance in different application fields. Si NW structures are increasingly attracting the attention of scientists with respect to key elements for optoelectronic device applications. One aspect that has been generally overlooked is the interaction of light of varying wavelengths with nanowire assemblies on a substrate, which is promising for future optical and optoelectronic applications. Sub-wavelength diameters and proximity effects combined with micron scale lengths may lead to interesting optical properties such as low reflectance and thus high absorption. Investigations of Sinanowire optical absorption have shown the size-dependent effect. ${ }^{1}$ Recently, photocurrent measurements have been used to qualitatively analyze the spectral absorption efficiency of n-Si NWs, grown from a substrate covered with the gold islands. ${ }^{2}$ It is shown that the maximum intensity of the photocurrent increases as the wavelength decreases and reaches a saturation regime below $375 \mathrm{~nm}$. By measuring the photoresponse of the photon energy, a shift of about $300 \mathrm{meV}$ towards a lower energy threshold for NW diameters increasing from $70 \mathrm{~nm}$ to $85 \mathrm{~nm}$ is found. Studies of the broadband optical absorption show increased total optical absorption spectra for

\footnotetext{
a) Author to whom correspondence should be addressed. Electronic mail: s.vitusevich@fz-juelich.de
}

Si NW samples. ${ }^{3} \mathrm{Si}$ NWs lead to a significant reduction of the reflectance compared to the solid film. ${ }^{3,4}$ Optical absorption increases while the wavelength decreases. Note that, unlike the bulk, the Si NW silicon may be direct band gap semiconductors, which would make them an excellent choice for optical applications. ${ }^{5-8}$ On the other hand, the energy bandgap increases while the nanowire width decreases. ${ }^{5}$ This may result in a successful shift of the absorption spectra to short wavelengths. Optical responses of boron-doped single Si NW-based metal-semiconductor-metal photodetectors are studied in Ref. 9, where high responsivity, of the order of $10^{4} \mathrm{~A} / \mathrm{W}$, is observed even at zero bias in a single NW photodetector with peak responsivity in the near-infrared region. The responsivity is found to increase with the increase of bias and decrease of NW diameter. For comparison, note that the unpackaged Si nanocrystal-based photodiode exhibits a peak of $0.02 \mathrm{~A} / \mathrm{W}$ in photoresponse to UV light in air. ${ }^{10}$ This is within an order of magnitude of the response of commercially available GaP-, GaN-, and SiC-based photodetectors. Calculations show that the maximal values of sensitivity at the $250 \mathrm{~nm}$ wavelength for photodiodes based on epitaxial silicon can reach values of up to $0.08-0.09 \mathrm{~A} / \mathrm{W} .^{11}$

On the other hand, sensing of the chemical and biological species is an essential area of research in fields such as diagnostics of diseases, screening, and development of new drugs or environmental monitoring. In terrestrial UV radiometers, the photodiode sensitivity is used within the wavelength range of 200 to $380 \mathrm{~nm}$. It is known that photodiodes based on silicon are sensitive in the near infrared spectral range. Si-based UV sensors are in great demand for military, security, and 


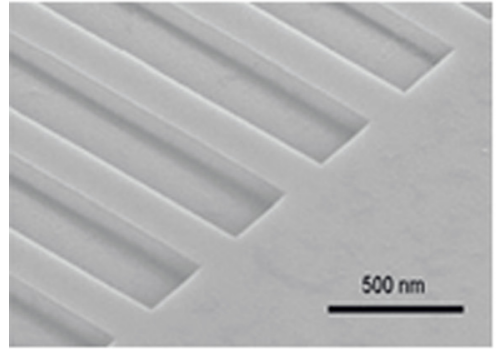

(a)

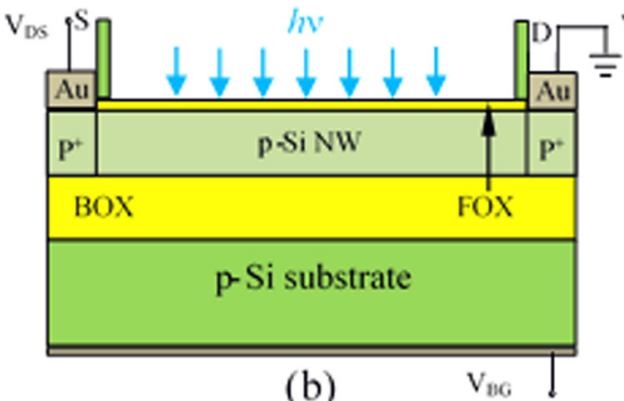

(b)

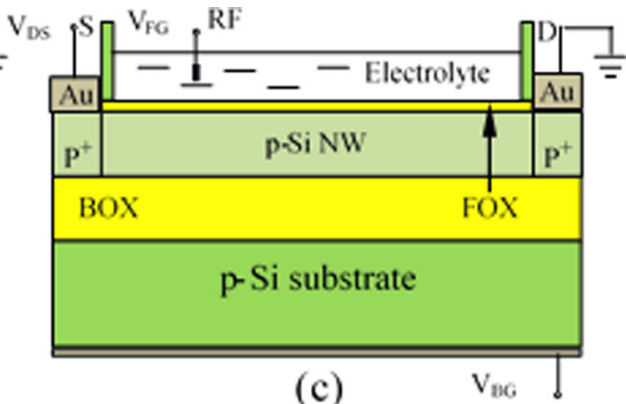

FIG. 1. Front $\mathrm{SiO}_{2}$; p-SiNW; buried $\mathrm{SiO}_{2}$; p-Si substrate FET structure: (a) SEM picture of the NWs, (b) schematic of the photoreceiver, and (c) schematic of the biochemical sensor. S, D, FOX, BOX, and RF defined source, drain, front oxide, buried oxide, and reference electrode, respectively.

commercial applications. Ion-sensitive field-effect transistors based on Si NW have also attracted substantial interest for various chemical and biochemical sensing applications yet uncertainty still remains concerning their response to changes in the supporting electrolyte concentration. ${ }^{12-15}$

The low-frequency (LF) noise ultimately limits the resolution of the sensor. Apart from the $\mathrm{pH}$ response, the evaluation of noise is also an important issue, as it ultimately defines the detection limit of a sensor. Recently, intense attempts have been made to understand the factors determining the signal-to-noise (SNR) ratio, ${ }^{15}$ however, with inconsistent conclusions about the optimal regime for sensing.

In this paper, the light absorption peculiarities and photosensitivity in the UV range of the spectrum and the detectivity of the Si NW $\mathrm{p}^{+}-\mathrm{p}-\mathrm{p}^{+}$FETs are investigated. In order to determine promising regimes for biosensing applications with optimal SNR, we performed both current-voltage (I-V) characteristics and noise measurements for solution-gated devices. We also investigated possibilities of LF-noise spectroscopy for liquid and back double-gated Si-NW-FET-based biochemical sensors. The analysis of the results of $\mathrm{pH}$ sensitivity and SNR of these structures allows important regimes to be identified for sensor utilization.

\section{SAMPLES AND EXPERIMENTAL TECHNIQUE}

Front oxide layer (FOX), p-Si NW, buried oxide layer (BOX), and p/Si substrate experimental devices (Fig. 1) are fabricated on the basis of SOI wafers (SOITEC). Thicknesses of the layers are $t_{F O X}=8 \mathrm{~nm}, t_{B O X}=145 \mathrm{~nm}, t_{N W}=50 \mathrm{~nm}$, the width of the nanowire is $250 \mathrm{~nm}$, and the length of the nanowire is $20 \mu \mathrm{m}$. The device has 50 identical parallel NWs. The detailed fabrication technique is described in Ref. 16. The concentration of acceptors (boron) in both substrate and $\mathrm{NW}$ is $10^{15} \mathrm{~cm}^{-3}$ and in $\mathrm{p}^{+}$source and drain regions $10^{19} \mathrm{~cm}^{-3}$. I-V characteristics, spectral dependences, and LF noise spectral density are measured on one type of NW FET structure at room temperature. The measurements of currentvoltage, and photoelectric and noise characteristics were performed using LabView program.

\section{PHOTOELECTRIC PROPERTIES AND DETECTIVITY}

The spectral photoresponse of Si NW FET structures is measured using monochromator YM-2. For the irradiation, we used incandescent lamps, positioned at $15 \mathrm{~cm}$ from the structure. NW samples are investigated in the wavelength range of $0.25-0.6 \mu \mathrm{m}$ at irradiation density $W=1.1 \mathrm{~W} / \mathrm{cm}^{2}$ and $1.6 \mathrm{~W} / \mathrm{cm}^{2}$, respectively. Fig. 2 shows the drain current, $I_{D}$, dependence on drain-source voltage, $V_{D S}$, measured in the dark and with light excitation. Spectral dependences of source-drain current, measured with and without light excitation, measured at $V_{B G}=-5 \mathrm{~V}$ and $V_{D S}=-5 \mathrm{~V}$, are shown in Fig. 3.

I-V characteristics show that the density of photoexcited carriers is small. As can be seen from Fig. 3, spectral dependences of the drain current are shifted to the short wavelength region compared to bulk Si samples. The results are in agreement with those reported in Ref. 2, where it is shown that a decrease in the nanowire diameter results in a spectral shift of the photocurrent intensity peak towards higher photon energies, which allows us to tune the absorption onset from the ultraviolet radiations to the visible light spectrum. Note that bulk Si is not (or only very slightly) sensitive in this part of the spectrum. In our Si NW samples, the drain current photosensitivity increases below wavelengths of $350 \mathrm{~nm}$ at $1.1 \mathrm{~W} / \mathrm{cm}^{2}$ irradiation and below $500 \mathrm{~nm}$ at $1.6 \mathrm{~W} / \mathrm{cm}^{2}$. The effect of spectral shift in Si NW (see Fig. 3) can be explained as follows:

- The NW width $(250 \mathrm{~nm})$ limits absorption of the long-wave photons.

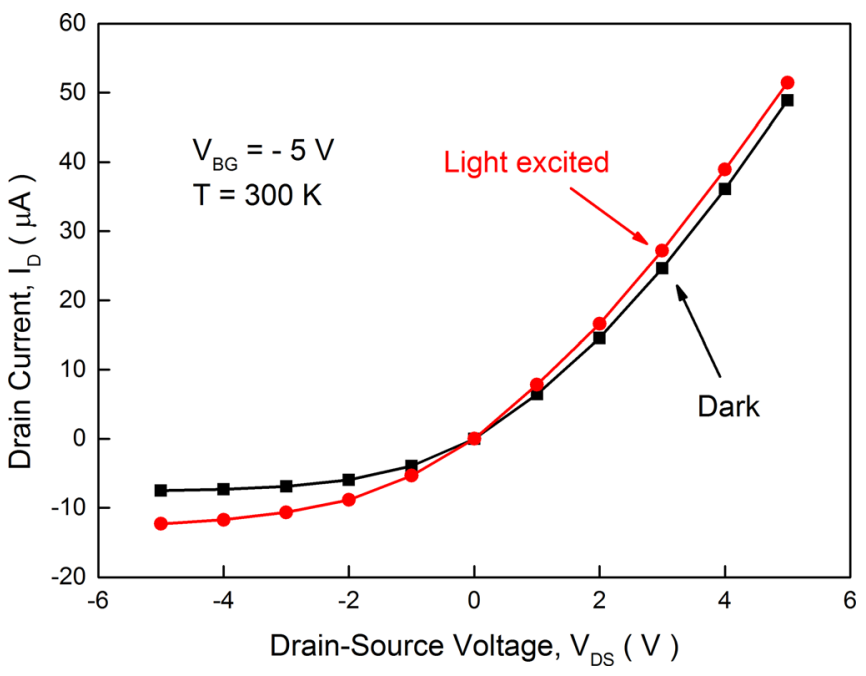

FIG. 2. Output current-voltage characteristics of Si NW FET sample, measured in the dark and with excitation by light at irradiation density $W=$ $1.1 \mathrm{~W} / \mathrm{cm}^{2}$. 


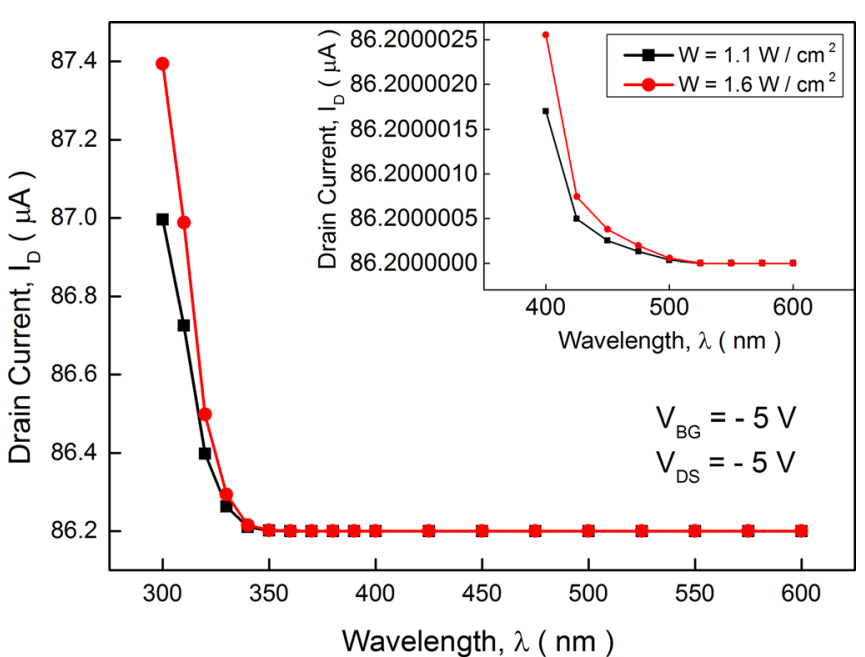

FIG. 3. Spectral dependences of photocurrent $I_{D}$ at irradiations, W/ $/ \mathrm{cm}^{2}: 1.1$ and 1.6. Inset: $I_{D}(\lambda)$ dependences for the wavelength range $400-600 \mathrm{~nm}$.

- As opposed to bulk silicon, the energy gap of the Si NWs increases while the NW size decreases. ${ }^{5}$

- Internal quantum yield for $\mathrm{Si}$ increases and becomes 2-3 at quantum energy $h \nu \geq 3 \mathrm{eV} .^{17}$

- As is known (see Ref. 18), the color of the emitted light is determined by the choice of the nanoparticle/quantum dot (QD) characteristic size $L_{Q D}$, since $h \nu=E_{g}+E_{e}+E_{h}$, where $E_{g}$ is the semiconductor bandgap energy, and the electron and hole confinement energies, $E_{e}$ and $E_{h}$, respectively. The energies increase with decreasing $L_{Q D}$. One can assume that the same explanation is also acceptable for NWs. According to the van Roosbroeck-Shockley relation, absorption and radiation are conjugating processes. ${ }^{19}$ The shift of the absorption spectra to the short-wave range can also be related to this fact. It is shown that these confinement (blue-shift) energies are proportional to $1 / L_{Q D}^{2} \cdot{ }^{18}$ With decreasing size, the short-wave photons can be more effectively absorbed in Si NWs.

The photosensitivity dependence as a function of drainsource voltage and several back-gate voltages, as well as spectral dependences of the photosensitivity, are shown in Figs. 4 and 5, respectively.

Sensitivity $R_{i}(\lambda)$ vs source-drain voltage and its spectral dependence was calculated according to

$$
R_{i}=\frac{\Delta I\left(V_{D S}\right)}{A W}, \quad R_{i}(\lambda)=\frac{\Delta I_{D}(\lambda)}{A W(\Delta \lambda)} .
$$

Here, $A$ is the photosensitive surface area of the NW, $\Delta I_{D}(\lambda)$ is the photocurrent at wavelength $\lambda, \Delta \lambda=10 \mathrm{~nm}$ is the minimal feasible wavelength range. It should be emphasized that values of the sensitivity vs $V_{D S}$ reach record values of 2-4 A/W at room temperature with increasing back-gate voltage. Sensitivity increases in the UV range of the spectrum and its values are about 10 times higher (see Figs. 4 and 5) than for bulk silicon photodetectors. ${ }^{20-24}$ For comparison, note that the internal responsivity $\sim 0.126 \mathrm{~A} / \mathrm{W}$ at a higher voltage $(8 \mathrm{~V})$ for a constant input optical power of $20 \mathrm{~mW}$ was demonstrated in graphene FET photodetectors. ${ }^{25}$ As opposed to our case, the detection mechanisms of the device investigated

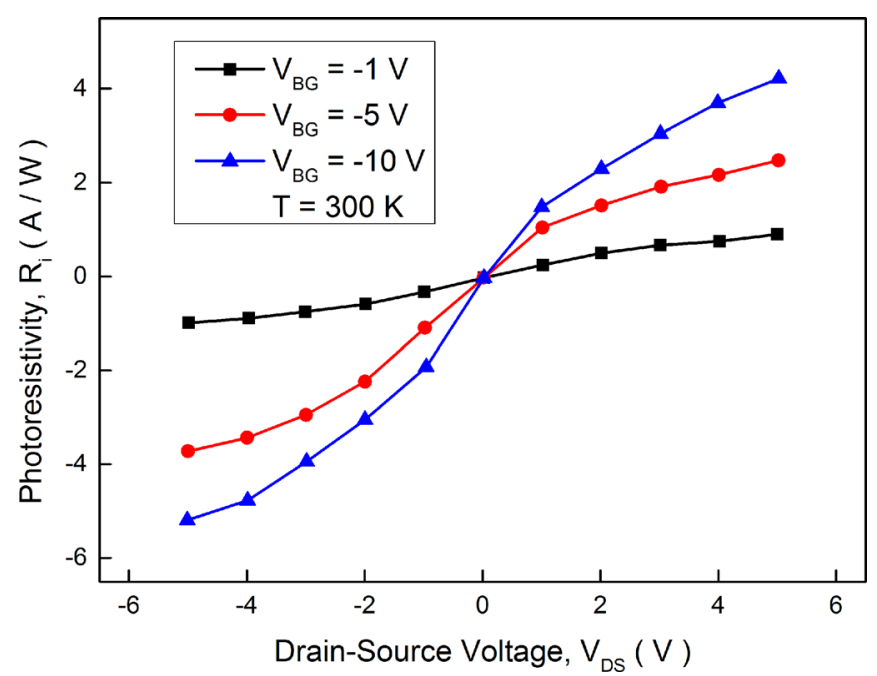

FIG. 4. Photosensitivity vs $V_{D S}$, obtained at integral illumination and at several back-gate voltages.

in Ref. 25 were based on the photo-thermoelectric and bolometric effects.

Detectivity, $D^{*}$, can be calculated from the following expression:

$$
\begin{aligned}
D^{*}(\lambda) & =\frac{\Delta I\left(V_{D S}, \lambda\right)}{\Delta I_{N}(f)} \frac{1}{A W} \sqrt{A \Delta f}=\frac{R_{i}\left(V_{D S}, \lambda\right)}{\Delta I_{N}(f)} \sqrt{A \Delta f} \\
& =\frac{R_{i}\left(V_{D S}, \lambda\right)}{\sqrt{S_{i}(f)}} \sqrt{A}=\frac{R_{i}\left(V_{D S}, \lambda\right)}{\sqrt{S_{V}(f)}} R \sqrt{A} .
\end{aligned}
$$

Here, $A$ is the area of the photosensitive region of the detector, $\Delta I_{N}(f)$ is the noise current, $S_{i}(f)$ is the current noise spectral density, $R$ is the Si NW resistance, and $\Delta f$ is the frequency bandwidth. Considering that the noise spectral density does not depend on irradiation wavelength, it is clear that the spectral dependence of the detectivity will show the same behavior as for sensitivity (Fig. 6). We used the data of the spectral photosensitivity (values of $R_{i}$ for corresponding

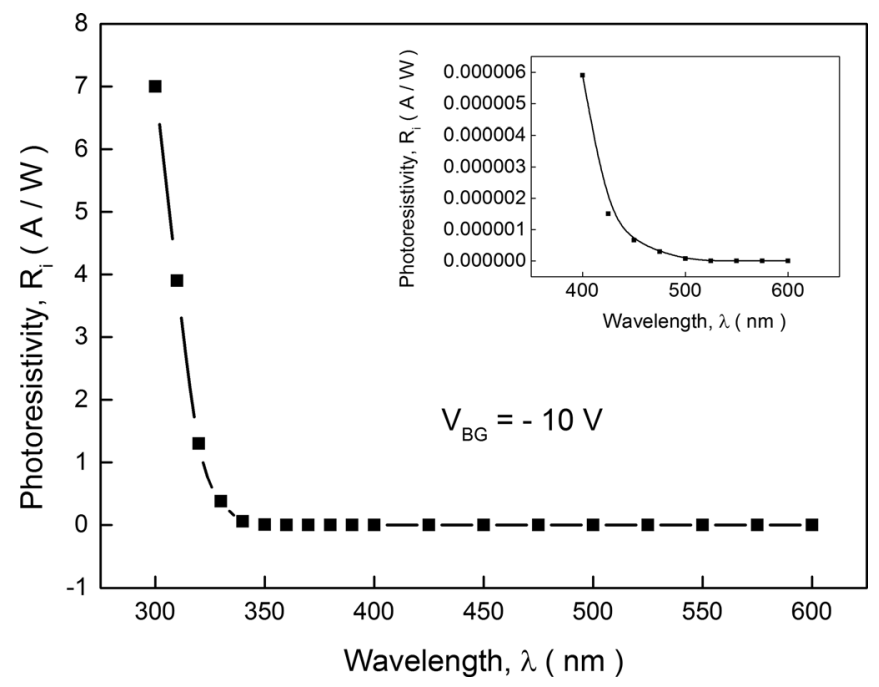

FIG. 5. Spectral dependence of photosensitivity at the $V_{D S}=-5 \mathrm{~V}$ and $V_{B G}=-10 \mathrm{~V}$. Inset shows part of spectral dependence in the wavelength range $400-600 \mathrm{~nm}$ on the enlarged scale. 

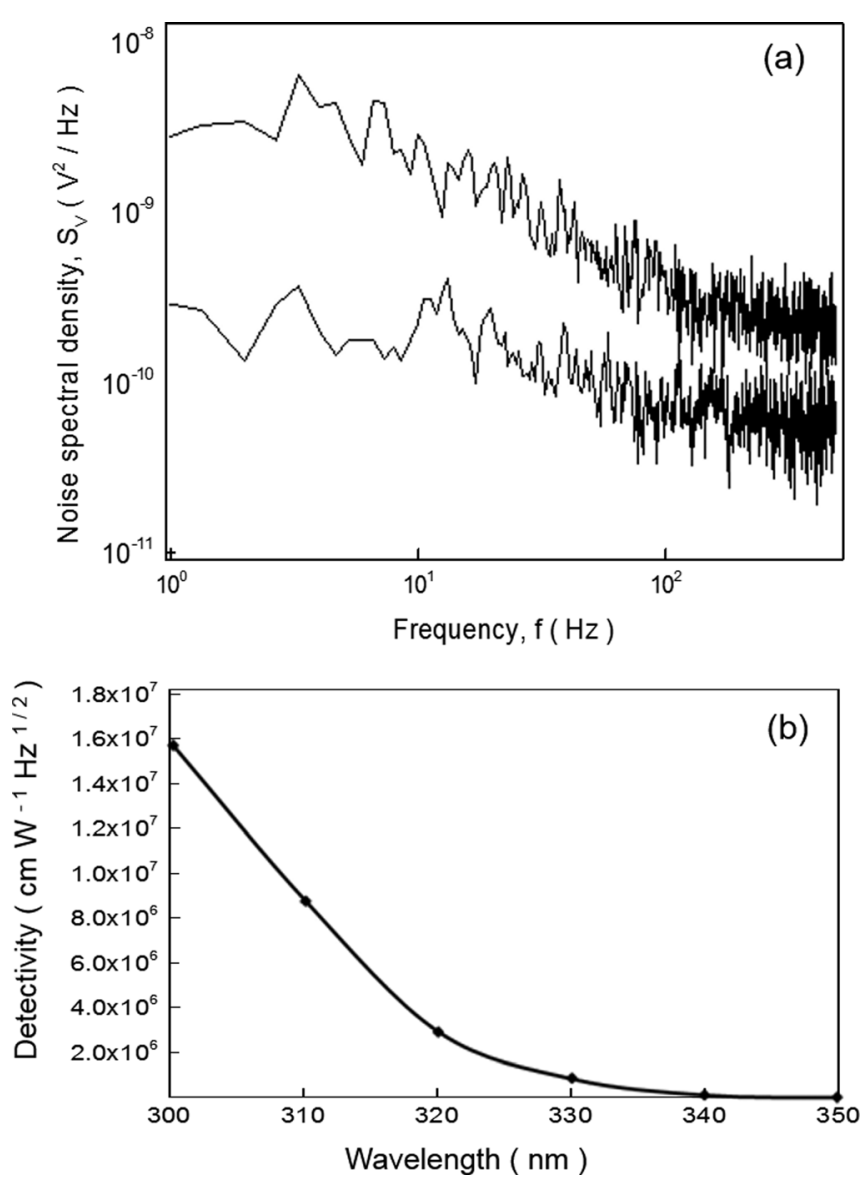

FIG. 6. (a) Spectral dependences of low-frequency noise, measured at irradiation power density $1.1 \mathrm{~W} / \mathrm{cm}^{2}$ as well as in the dark at $300 \mathrm{~K}$; (b) spectral dependence of detectivity at irradiation power density $1.1 \mathrm{~W} / \mathrm{cm}^{2}$, $V_{D S}=-5 \mathrm{~V}, V_{B G}=-10 \mathrm{~V}$, and $300 \mathrm{~K}$.

wavelength, $\lambda$, and $V_{B G}$ ) from Fig. 5 at resistance $r=1.3$ $\times 10^{5} \Omega$ in the linear region of the $\mathrm{I}-\mathrm{V}$ characteristic and measured spectral dependences of low-frequency noise in the dark and under irradiation (shown in Fig. 6(a)) for plotting $D^{*}(\lambda)$ dependence (Fig. 6(b)).

The values of the detectivity increase with decreasing wavelength. This fact is promising for biosensor applications.

\section{SIGNAL-TO-NOISE RATIO (SNR) AND pH SENSITIVITY}

Over the past decades, interfaces between oxides and electrolytes have received considerable attention in different scientific communities. Detection and quantitation of biological species are central to many areas in healthcare. The signal-to-noise ratio (SNR) is the key metric describing sensor performance. Noise spectroscopy and diagnostics are among the most powerful and sensitive tools for studying the properties of nanosize semiconductors and multi-purpose devices. The phenomenon of the low-frequency (LF) fluctuation of electric current in semiconductors and semiconductor devices is the subject of investigations by many researchers and engineers. ${ }^{26-31}$ Studies have shown that the behavior and magnitude of LF noise in devices based on nanosize silicon structures differs essentially from the noise in devices based on crystalline silicon. ${ }^{27-30}$
The SNR was analyzed to estimate the signal enhancement and possible detection limit. The SNR for Si NW FETs operated in an electrolyte environment is an essential figure of merit for characterizing and comparing the detection limit of such devices used in an exposed channel configuration as biochemical sensors. At a later date, we employ LF-noise measurements to determine the regime for optimal SNR. In the case of liquid-gated FET sensors, the signal (response) is given by a change in surface potential or drain current upon changing the bulk concentration of the targeted analyte.

Noise spectroscopy and transconductance measurements establish the optimal operating regimes for Si NW FET sensors employed in Ref. 16. A strong coupling between the liquid gate and back gate has been revealed and used for the optimization of SNR in sub-threshold as well as abovethreshold regimes. The fabrication technique for liquid-gated Si NW FETs and their transport and dynamic properties are investigated experimentally and theoretically in detail in Ref. 32. Double-gated Si NW FETs were fabricated, and their pH-sensing capabilities were also investigated in Ref. 33. The proposed Si NW FETs allow the gate voltage to be addressed independently and hence improve the sensing capability through an application of asymmetric gate voltages. One gate is a driving gate which controls the current flow, and the other is a supporting gate which amplifies the shift of the threshold voltage, $V_{T}$, which is a sensing metric, and which arises from changes in the $\mathrm{pH}$. The $\mathrm{pH}$ response was defined as the amount of $V_{T}$ shift when the $\mathrm{pH}$ in the injected solution was varied from $\mathrm{pH}=4$ to $\mathrm{pH}=10$.

It is known that the normalized current noise power amplitude $\left(S_{I} / I^{2}\right.$ or $\left.S_{V} / V^{2}\right)$ reaches a plateau and is highest in the sub-threshold regime for $\mathrm{Si} / \mathrm{SiO}_{2}$ devices, ${ }^{34,35}$ and concerns have been expressed that SNR would be impacted for measurements carried out in the sub-threshold. ${ }^{36,37}$ On the other hand, $S_{I} / I^{2}$ is lower in the linear regime but the sensitivity is also lower. The combination of Si NW FET sensors with rolling circle amplification (RCA) will increase diagnostic capacity and the ability of laboratories to detect unexpected viruses, making it a potential tool for the early diagnosis of gene-related diseases. A novel approach for rapid, label-free and specific DNA detection by applying RCA based on $\mathrm{Si}$ NW FET is described in Ref. 38. In Ref. 39, it is shown that SNR is not significantly affected by the electrolyte concentration, composition, or $\mathrm{pH}$.

We studied the $\mathrm{pH}$ sensitivity and signal-to-noise ratio (SNR) of the Si-NW-FET-based biochemical sensors, shown schematically in Fig. 1(c). Noise spectral density is measured in the frequency range of $2-500 \mathrm{~Hz}$ at several values of the electrolyte $\mathrm{pH}$ and liquid-gated voltages. A technique similar to that described in Ref. 40 is used for noise measurements.

Measurements of I-V characteristics are performed in an aqueous solution with different $\mathrm{pH}$ values. The drain current hardly depends on the $\mathrm{pH}$ value (typical $\mathrm{I}-\mathrm{V}$, measured at $\mathrm{pH}=7$ is shown in Fig. 7(a)). Dependences of the drain current vs. liquid-gate voltage at several $\mathrm{pH}$, at zero back-gate voltage, and $V_{D S}=-1 \mathrm{~V}$ are shown in Fig. 7(b).

The drain current displays behavior in agreement with Refs. 16 and 32 and grows when the $\mathrm{pH}$ value increases. Such behavior can be explained as follows. As is shown in 
(a)

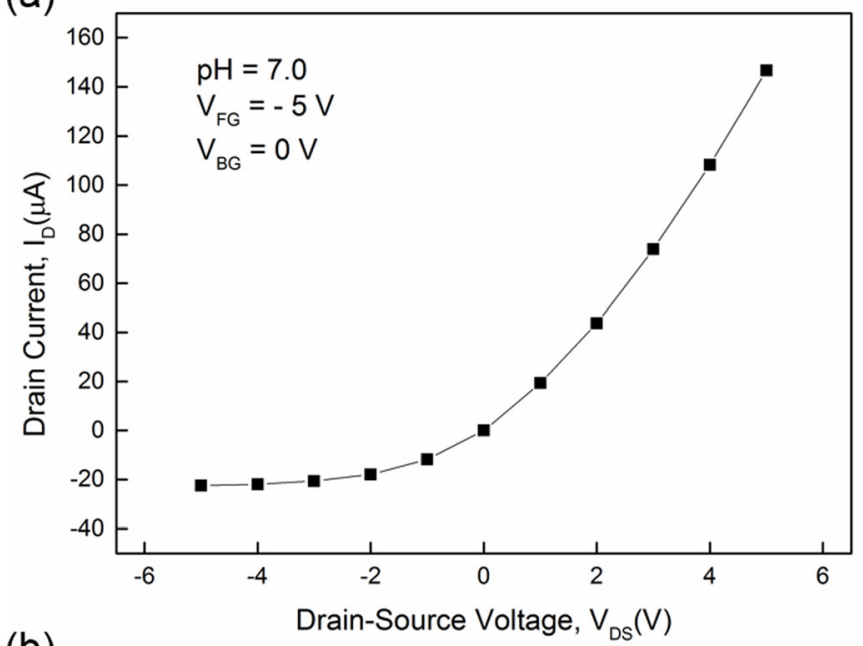

(b)

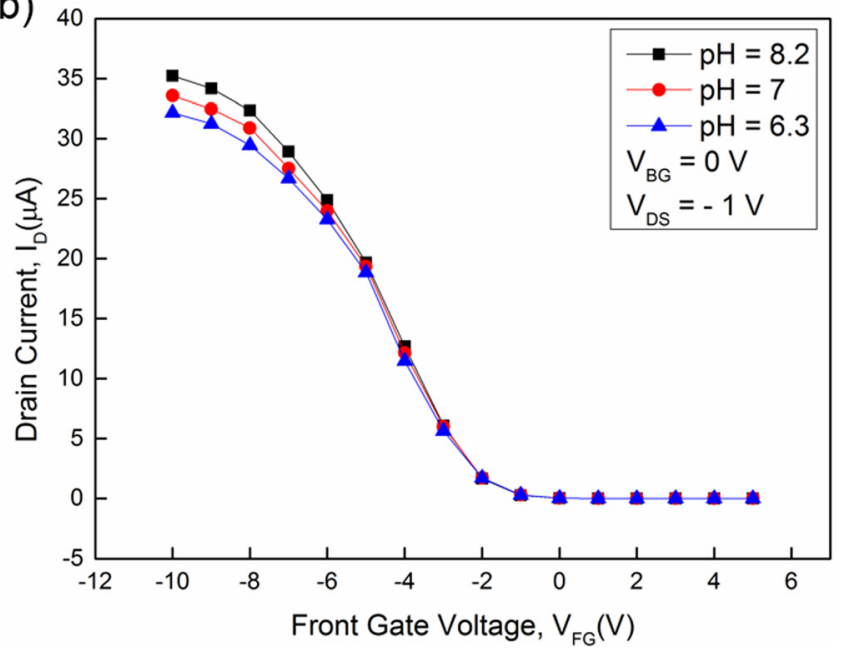

FIG. 7. (a) Output I-V characteristic at $\mathrm{V}_{\mathrm{FG}}=-5 \mathrm{~V}, \mathrm{~V}_{\mathrm{BG}}=0 \mathrm{~V}$, and $\mathrm{pH}=7$; (b) drain current as a function of liquid gate voltage, measured at different $\mathrm{pH}$ values.

Refs. 9 and 32, in the FOX layer, there are repulsive character traps located near the $\mathrm{Si} / \mathrm{SiO}_{2}$ interface. Then under the influence of these repulsive traps, the energy bandgap of the p-type Si NW bends in the interfacial region and a depletion region is formed. The size of this region, $L_{0}$, is equal to

$$
L_{0}=\sqrt{\frac{2 \varepsilon \varepsilon_{0}\left(\chi_{o x}-\chi_{S i}\right)}{e^{2} p}} .
$$

Here, $\varepsilon$ and $\varepsilon_{0}$ are the silicon and free-space permittivity, respectively; $\chi_{o x}$ and $\chi_{S i}$ are the affinity energies of the front oxide $\left(\mathrm{SiO}_{2}\right)$ and silicon, respectively; $p$ is the concentration of the majority carriers in silicon. Taking into account that all shallow acceptors in $\mathrm{p}-\mathrm{Si} \mathrm{NW}$ are ionized at room temperature, we can assume that $p=N_{A}=10^{15} \mathrm{~cm}^{-3}$. Then, we consider that nanowire length $L=2 \mu \mathrm{m}$, is about two orders larger than the NW thickness of $50 \mathrm{~nm}$. It can be seen that the current channel in p-Si NW is completely depleted and has high resistance. At $V_{F G}=0 \mathrm{~V}$, the channel is closed and the drain current is absent. With the growth of $V_{F G}\left(V_{F G}<0\right)$, the channel begins to open and the drain current increases. The drain current is the signal current by means of which we receive information about the sensitivity of electrolyte composition and charged ion concentration ( $\mathrm{pH}$ value) contained in it. The level of the useful signal is limited by the internal noise, and particularly, by the LF noise since adsorption-desorption processes on the electrolyte-oxide interface are very slow. The measured data are in good agreement with the trap state model, assuming trapping/detrapping of the trap states at the $\mathrm{Si} / \mathrm{SiO}_{2}$ interface as one of the sources of the LF noise.

Fig. 8 shows the spectral dependence of the lowfrequency noise, measured at different values of the liquid gate voltage at $300 \mathrm{~K}$.

As is known, in the electrolyte-insulator-semiconductor structure noise can also be formed by the random tunneling of the majority carriers from the channel to electrolyte. Probability of tunneling occurs when the potential barrier height (in our case front-oxide thickness, $8 \mathrm{~nm}$ ) is less than or equal to the de Broglie wavelength of the majority carriers (in $\mathrm{Si}$ it is equal to $\sim 5 \mathrm{~nm}$ (Ref. 41)). Then, the tunneling probability is very low, as will be shown below. In this case, for the description of low-frequency noise we can use the correlated number-mobility fluctuation unified $\Delta n-\Delta \mu$ model. According to this model, the flicker noise spectral density can be presented as follows: ${ }^{42}$

$$
S_{V_{D S}}(f)=\frac{e^{2} k T}{\gamma f w L C_{o x}^{2}}\left(1+\alpha \mu_{h} p\right)^{2} N_{t}\left(E_{f}\right) .
$$

Here, $w$ and $L$ are the channel width and length, respectively; $C_{o x}$ is the capacitance of the insulator layer $\left(\mathrm{SiO}_{2}\right), \gamma=$ $4 \pi \sqrt{2 m_{o x}^{*} \phi_{B}} / h$ is the tunneling parameter (for $\mathrm{Si}_{-} \mathrm{SiO}_{2}$ it is equal to $10^{8} \mathrm{~cm}^{-1}$ (Ref. 43)), $\phi_{B}$ is the tunneling barrier height for holes at the interface, $m_{o x}^{*}$ is the holes effective mass in the oxide layer, $\alpha=\pi m^{*} e^{2} / 4 h k T\left(\varepsilon_{S i}+\varepsilon_{O X}\right)^{2}$ is the carrier density function formed by the screening effect, $m^{*}$ is the hole effective mass in the silicon, $\mu_{h}$ is the hole mobility, $N_{t}\left(E_{f}\right)$ is the energetic distribution of the traps, and $E_{f}$ is the Fermi energy.

As follows from Eq. (4), $S_{V_{D S}} \propto p^{2}$. The curve presented in Fig. 9 provides evidence in favor of the application of the unified $\Delta n-\Delta \mu$ model to describe the noise, showing the

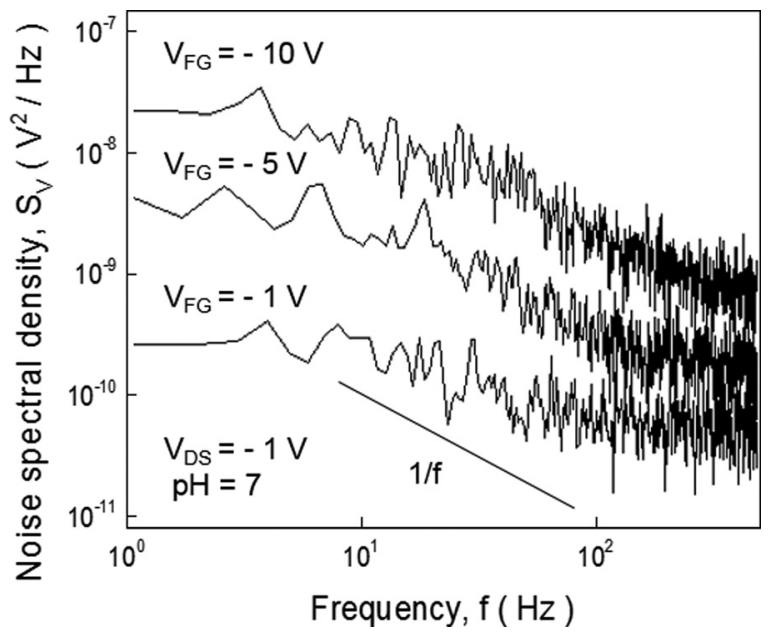

FIG. 8. Noise spectra, measured at different values $\mathrm{V}_{\mathrm{FG}}$ for $\mathrm{pH}=7$. 


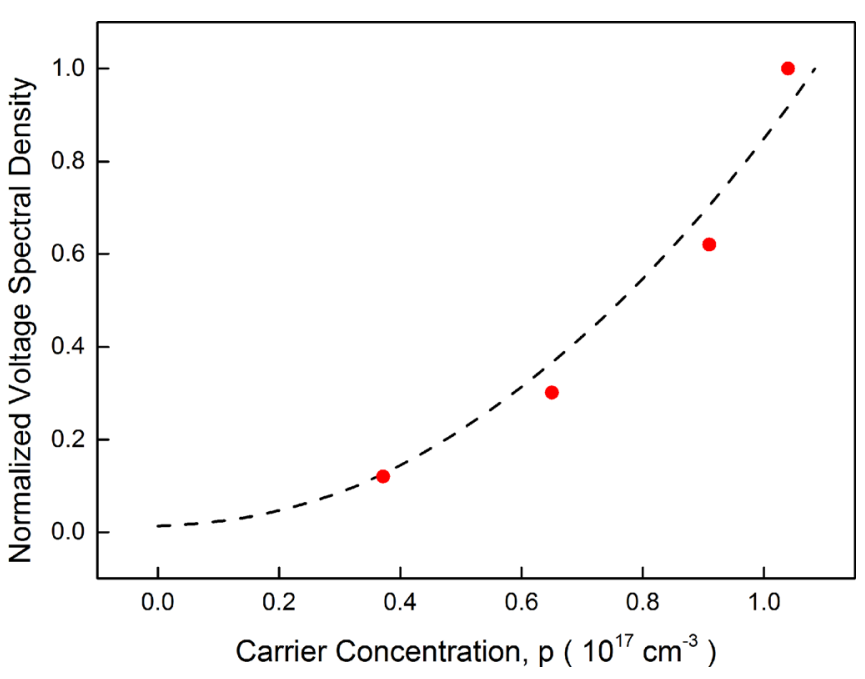

FIG. 9. Normalized noise dependence vs. carrier concentration.

dependence of the normalized noise vs. the concentration of the majority carriers.

This is a quadratic dependence on concentration. Values for the $p$ plotted on axis $\mathrm{X}$ in Fig. 9 are calculated for the linear part of the I-V characteristic ${ }^{43}$

$$
I_{D}=\frac{e p \mu_{h} W V_{D S} t_{N W}}{L} .
$$

In order to plot Fig. 9, according to Eq. (4), the values of the concentration of the majority carriers $p$ are calculated from Eq. (5) at $w=250 \mathrm{~nm}, \mu=130 \mathrm{~cm}^{2} / \mathrm{V} \mathrm{s}$ (see also Ref. 44 ), $L=20 \mu \mathrm{m}$, and $I_{D}=0.1 \mu \mathrm{A}$ (linear part of I-V characteristics). It is clear that having measured the dependences of noise spectral density we can determine the concentration of the majority carriers in the nanosize channel. The LF noise spectral density behavior can be explained by the following:

1. As is known, quantization effects in Si NW lead to the shift of the maximum values of the concentration of the majority carriers from the $\mathrm{Si} / \mathrm{SiO}_{2}$ interface. ${ }^{16}$ The majority carriers are located near FOX and occupy the region at a depth of 1-2 nm. More than $90 \%$ of the channel thickness does not contribute to the dynamic processes and is in a passive state. Carriers concentrated near the FOX layer will influence and increase the probability of tunneling and the adsorption-desorption processes of the ionized particles will take place more intensively on the $\mathrm{Si} / \mathrm{SiO}_{2}$ interface. On the other hand, high values of the electron capture time slope $(-2.8)$ in $\mathrm{p}-\mathrm{Si} \mathrm{NW}$ and its strong dependence on drain current ${ }^{32}$ can also influence the intensity of adsorption-desorption processes. These factors reflect physical processes in the structure.

2. It is known that one of the main mechanisms of flicker noise origin in semiconductor media is related to the interactions between carrier and acoustic phonon systems. ${ }^{45}$ Carrier distribution in the channel is strongly nonuniform due to the quantization effect, which results in greater disturbance of the thermodynamic equilibrium between holes and the acoustic phonon system in Si NW and increased fluctuations in the structure current.
It is known that the $\mathrm{pH}$ sensitivity of biochemical sensors is determined by the changing of the semiconductor surface potential, which directly influences the change in $I_{D}$. Then, the $\mathrm{pH}$ sensitivity $R_{p H}$ can also be determined as follows:

$$
R_{p H}=\frac{\Delta I_{D}}{\Delta p H} .
$$

As is shown in Fig. 10, $\mathrm{pH}$ sensitivity increases with increasing liquid gate voltage and tends to saturation $\left(R_{p H, s a t} \approx 0.455 \mu \mathrm{A} / \mathrm{pH}\right)$.

Note that $\mathrm{pH}$ sensitivity of the drain current of Si-NWFET-based $\mathrm{pH}$-sensors as a function of liquid gate voltage has not been reported in the literature. At high values of $V_{F G}$, the sensitivity tends to saturation value, because at high values of the $V_{F G}$ almost all carriers in the channel will take part in the drain current. It should be noted that current sensitivity can be made more efficiently and experimentally accessible. For comparison, note that for similar sensors, a maximum Nernstian sensitivity to $\mathrm{pH}$ change of $59 \mathrm{mV} / \mathrm{pH}^{46}$ and $59.5 \mathrm{mV} / \mathrm{pH}$ (Nernst limit) ${ }^{47}$ was obtained experimentally. As follows from Fig. 2, in the linear part of the I-V characteristic at $V_{D S}=1 \mathrm{~V}$ for electrical resistance, we have $r \approx 1.3 \times 10^{5} \Omega$. Then for our sensors, the maximal value of the voltage $\mathrm{pH}$ sensitivity will be equal to

$$
\begin{aligned}
R_{p H, V} & =r \times R_{p H, s a t}=1.3 \times 10^{5} \Omega \times 0.455 \mu \mathrm{A} / \mathrm{pH} \\
& =59.15 \mathrm{mV} / \mathrm{pH} .
\end{aligned}
$$

This value for $\mathrm{pH}$ sensitivity is very close to the Nernst limit.

Fig. 11 shows the signal-to-noise ratio (SNR) dependences on liquid gate voltage at different frequencies.

The SNR is calculated according to the following expression: ${ }^{16}$

$$
S N R=\frac{\Delta V_{F G_{\text {Signal }}}}{\delta V}=\frac{\Delta V_{F G_{\text {Signal }}}}{\sqrt{S_{V} \Delta f}} .
$$

Here, $\Delta V_{F G_{\text {Signal }}}$ is the signal value of the liquid gate voltage, $\delta V$ is the root-mean-square (rms) voltage noise amplitude

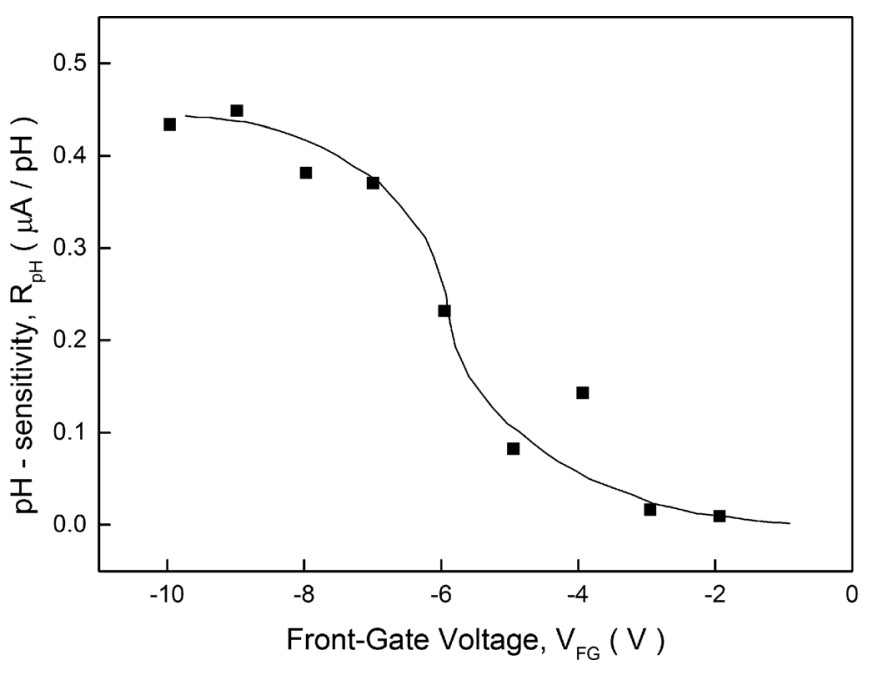

FIG. 10. pH sensitivity dependence on liquid-gate voltage. 


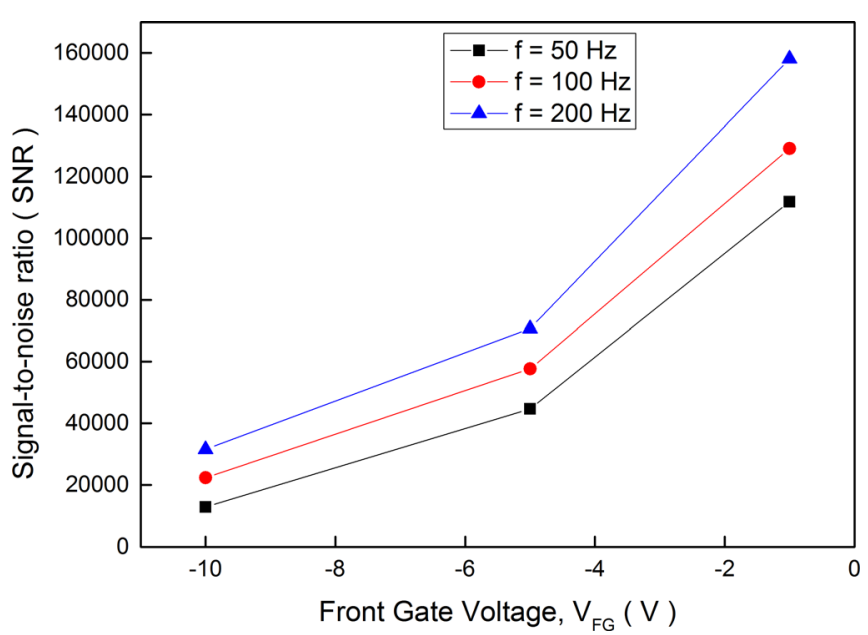

FIG. 11. Signal-to-noise ratio dependence on liquid-gate voltage at several frequencies for gate signal voltage $\Delta \mathrm{V}_{\mathrm{FG}}$, Signal $=1 \mathrm{~V}$.

( $\delta V$ is obtained from $S_{V}$ ), and $\Delta f=1 \mathrm{~Hz}$ is the frequency unit interval. For the numerical calculation, we take $\Delta V_{F G_{\text {Signal }}}=1 \mathrm{~V}$.

At low values of the liquid gate voltages, SNR can reach very high values $\sim 10^{5}$. The obtained results have to be taken into account for designing high-sensitivity biosensors.

\section{CONCLUSION}

I-V characteristics, photosensitivity, and detectivity of $\mathrm{p}^{+}-\mathrm{p}-\mathrm{p}^{+}$Si NW FETs are investigated. It is shown that the absorbance of p-Si NW shifts to the short-wave region and compared with bulk Si photocurrent the photosensitivity reaches higher values in the UV range of the spectrum at $300 \mathrm{~K}$. Therefore, Si NW FETs can be successfully used as UV photodetectors, including for biosensor applications.

For Si NW sensors, transport and noise properties were analyzed, as well as $\mathrm{pH}$ sensitivity behavior as a function of the liquid gate voltage. We revealed that $\mathrm{pH}$ sensitivity of the drain current of Si NW FET-based pH sensors increased with applied liquid gate voltage at $300 \mathrm{~K}$. A strong dependence of $\mathrm{pH}$ sensitivity, as a result of the liquid gate voltage increase, gave us the opportunity to measure very low proton concentrations in the electrolyte medium at the comparatively high values of the liquid gate voltage. Low-frequency noise is analyzed. Using noise spectroscopy, it is possible to determine the concentration of the majority carriers in the nanosize channel according to Eq. (4). The signal-to-noise ratio for $\mathrm{Si}-\mathrm{NW}$-based biochemical sensors displays a high value, reaching up to $10^{5}$. The noise characteristic index decreases from 1.1 to 0.7 with increasing $V_{F G}$ value. Noise behavior is successfully explained in the framework of the correlated number-mobility fluctuation unified model.

\section{ACKNOWLEDGMENTS}

F. Gasparyan greatly appreciates the support from the German Academic Exchange Service (DAAD) in the form of a research grant. This work was supported by the German Federal Ministry of Education and Research (BMBF Project
No. 01DK13016). This work was also supported by the RA MES State Committee of Science, in the framework of research Project No. 15T-1C 279. We thank Dr. Stefan Trellenkamp for electron beam writing and the technological staff of the Helmholtz Nanoelectronic Facility for assistance in device fabrication at Forschungszentrum Jülich. The authors are grateful to the anonymous referees for their useful and constructive criticism.

${ }^{1}$ G. Chen and L. Hu, "Silicon nanowires for solar photovoltaic applications," SPIE Newsroom (2008).

${ }^{2}$ T. Xu, Y. Lambert, Ch. Krzeminski, B. Grandidier, D. Stiévenard, G. Lévêque, A. Akjouj, Y. Pennec, and B. Djafari-Rouhani, "Optical absorption of silicon nanowires,” J. Appl. Phys. 112, 033506 (2012).

${ }^{3}$ L. Tsakalakos, J. Balch, J. Fronheiser, M.-Y. Shih, S. F. LeBoeuf, M. Pietrzykowski, P. J. Codella, B. A. Korevaar, O. Sulima, J. Rand, A. Davuluru, and U. Rapolc, "Strong broadband optical absorption in silicon nanowire films," J. Nanophotonics 1, 013552 (2007).

${ }^{4}$ E. Garnett and P. Yang, "Light trapping in silicon nanowire solar cells," Nano Lett. 10, 1082-1087 (2010).

${ }^{5}$ V. Parkash and A. K. Kulkarni, "Optical absorption characteristics of silicon nanowires for photovoltaic applications," IEEE Trans. Nanotechnol. 10(6), 1293-1207 (2011).

${ }^{6} \mathrm{G}$. Sanders and Y. C. Chang, "Theory of optical properties of quantum wires in porous Si," Phys. Rev. B 45, 9202-9213 (1992).

${ }^{7}$ A. Miranda, R. Vázquez, A. Díaz-Méndez, and M. Cruz-Irisson, "Optical matrix elements in tight-binding approach of hydrogenated Si nanowires," Microelectron. J. 40(3), 456-458 (2009).

${ }^{8}$ M. Bruno, M. Palummo, S. Ossicini, and R. D. Sole, "First-principles optical properties of silicon and germanium nanowires," Surf. Sci. 601(13), 2707-2711 (2007).

${ }^{9}$ K. Das, S. Mukherjee, S. Manna, S. K. Ray, and A. K. Raychaudhuri, "Single Si nanowire $($ diameter $\leq 100 \mathrm{~nm})$ based polarization sensitive near-infrared photodetector with ultra-high responsivity," Nanoscale 6, $11232-11239$ (2014).

${ }^{10}$ T. Lin, X. Liu, B. Zhou, Z. Zhan, A. N. Cartwright, and M. T. Swihart, "A solution-processed UV-sensitive photodiode produced using a new silicon nanocrystal ink," Adv. Funct. Mater. 24(38), 6016-6022 (2014).

${ }^{11}$ Yu. Dobrovolskyi, L. Pidkamin, V. Brus, and V. Kuzenko, "Photodiode based on epitaxial silicon with high sensitivity at the wavelength $254 \mathrm{~nm}$," Semicond. Phys., Quantum Electron. Optoelectron. 17(2), 256-259 (2014).

${ }^{12}$ Y. Cui, Q. Wei, H. Park, and C. M. Lieber, "Nanowire nanosensors for highly sensitive and selective detection of biological and chemical specie," Science 293, 1289-1292 (2001).

${ }^{13}$ E. Stern, J. F. Klemic, D. A. Routenberg, P. N. Wyrembak, D. B. TurnerEvans, A. D. Hamilton, D. A. LaVan, T. M. Fahmy, and M. A. Reed, Nature 445, 519-522 (2007).

${ }^{14} \mathrm{O}$. Knopfmacher et al., "Nernst limit in dual-gated Si-nanowire FET sensors," Nano Lett. 10, 2268-2274 (2010).

${ }^{15}$ A. Tarasov, "Silicon nanowire field-effect transistors for sensing applications," PhD thesis (Basel, 2012).

${ }^{16}$ S. Pud, J. Li, V. Sibiliev, M. Petrychuk, V. Kovalenko, A. Offenhäusser, and S. Vitusevich, "Liquid and back gate coupling effect: Toward biosensing with lowest detection limit," Nano Lett. 14, 578-584 (2014).

${ }^{17}$ V. S. Vavilov, Action of Irradiation on the Semiconductors (Fizmatgiz, Moscow, 1963) (in Russian).

${ }^{18}$ E. L. Wolf, Nanophysics and Nanotechnology: An Introduction to Modern Concepts in Nanoscience, 2nd ed. (Wiley-VCH Verlag GmbH \&Co. KGaA, Weinheim, 2006).

${ }^{19}$ J. I. Pankove, Optical Processes in Semiconductors (Prentice-Hall, New Jersey, 1971).

${ }^{20}$ See http://www.labsphere.com/products/spheres-and-components/laserpower-measurement-spheres/detector-assemblies.aspx for information about sensitivity of bulk silicon photodetectors of Labsphere company.

${ }^{21}$ See https://www.solarmeter.com/model57.html for information about sensitivity of "Solarmeter Model 5.7" bulk silicon photodetectors.

${ }^{22}$ See http://www.kyosemi.co.jp/en/sensor/gan_uv_sensor/kpdu37s1_q1 for information about sensitivity of bulk silicon photodetectors of Kyosemi company. 
${ }^{23}$ See http://physics.nist.gov/Pubs/TN1421/detector.html for information about sensitivity of bulk silicon photodetectors of NIST Physical Measurement Laboratory.

${ }^{24} \mathrm{See} \quad$ http://www.scitec.uk.com/uvphotodiodes/uvphotodiodes/notes/uv_ index_measuring for information about sensitivity of bulk silicon photodetectors of Scitec Instruments company.

${ }^{25}$ J. Wang, Z. Cheng, Z. Chen, J.-B. Xu, H. Ki Tsang, and C. Shu, J. Appl. Phys. 117, 144504 (2015).

${ }^{26} \mathrm{~F}$. V. Gasparyan, "Noise reduction in (bio-) chemical sensors functionalized with carbon nanotube multilayers," in Advanced Sensors for Safety and Security, NATO Science for Peace and Security Series B: Physics and Biophysics, edited by A. Vaseashta and S. Khudaverdyan (Springer Netherlands, 2013), Chap. 11, pp. 139-150.

${ }^{27}$ S. Vitusevich and F. Gasparyan, "Low-frequency noise spectroscopy at nanoscale: Carbon nanotube materials and devices," in Carbon Nanotubes Applications on Electron Devices, edited by J. M. Marulanda (InTech, 2011), Chap. 11, pp. 257-296.

${ }^{28}$ S. Pud, F. Gasparyan, M. Petrychuk, J. Li, A. Offenhäusser, and S. Vitusevich, "Single trap dynamics in electrolyte-gated Si-nanowire field effect transistors," J. Appl. Phys. 115, 233705(1-11) (2014).

${ }^{29}$ F. V. Gasparyan, A. Poghossian, S. A. Vitusevich, M. V. Petrychuk, V. A. Sydoruk, J. R. Siqueira, Jr., O. N. Oliveira, A. Offenhäusser, and M. J. Schöning, "Low-frequency noise in field-effect devices functionalized with dendrimer/carbon- nanotube multilayers," IEEE Sens. J. 11(N1), 142-149 (2011)

${ }^{30} \mathrm{~F}$. Gasparyan, "Low-frequency noises in nanotubes and nanowires," Arm. J. Phys. 3(4), 312-341 (2010).

${ }^{31}$ F. V. Gasparyan, "Excess noises in (bio-)chemical nanoscale sensors," Sens. Transducers J. 122(11), 72-84 (2010).

${ }^{32}$ F. Gasparyan, I. Zadorozhnyi, and S. Vitusevich, "Single trap in liquid gated nanowire FETs: Capture time behavior as a function of current," J. Appl. Phys. 117, 174506-1 (2015).

${ }^{33}$ J.-H. Ahn, J.-Y. Kim, M.-L. Seol, D. J. Baek, Z. Guo, Ch.-H. Kim, S.-J. Choi, and Y.-K. Cho, "A pH sensor with a double-gate silicon nanowire field-effect transistor," Appl. Phys. Lett. 102, 083701 (2013).

${ }^{34}$ G. Ghibaudo, O. Roux, C. Nguyenduc, F. Balestra, and J. Brini, Phys. Status Solidi A 124(2), 571 (1991).
${ }^{35}$ J. Zhuge, R. S. Wang, R. Huang, Y. Tian, L. L. Zhang, D. W. Kim, D. Park, and Y. Y. Wang, IEEE Electron Device Lett. 30(1), 57 (2009).

${ }^{36}$ Y. Cheng, P. Xiong, C. S. Yun, G. F. Strouse, J. P. Zheng, R. S. Yang, and Z. L. Wang, Nano Lett. 8(12), 4179 (2008).

${ }^{37}$ Y. M. Lin, J. Appenzeller, J. Knoch, Z. H. Chen, and P. Avouris, Nano Lett. 6(5), 930 (2006).

${ }^{38}$ A. Gao, N. Zou, P. Dai, N. Lu, T. Li, Y. Wang, J. Zhao, and H. Mao, "Signal-to-noise ratio enhancement of silicon nanowires biosensor with rolling circle amplification," Nano Lett. 13(9), 4123-4130 (2013).

${ }^{39}$ N. K. Rajan, D. A. Routenberg, and M. A. Reed, "Optimal signal-to-noise ratio for silicon nanowire biochemical sensors," Appl. Phys. Lett. 98, 264107(1-3) (2011).

${ }^{40} \mathrm{~F}$. Gasparyan, H. Khondkaryan, and M. Aleksanyan, "New application of the noise spectroscopy for hydrogen sensors," J. Mod. Phys. 5, 1662-1669 (2014).

${ }^{41}$ A. Sacchetti, "Electrical current in nanoelectronic devices," Phys. Lett. A 374(10), 4057-4060 (2010).

${ }^{42}$ K. K. Hung, P. K. Ko, P. C. Hu, and Y. C. Cheng, "A unified model for the Flicker noise in metal-oxide-semiconductor field-effect transistors," IEEE Trans. Electron Devices 37(3), 654-665 (1990).

${ }^{43} \mathrm{H}$. Tian and A. El Gamal, "Analysis of 1/f noise in switched MOSFET circuits," IEEE Trans. Circuits Systems, II: Analog Digital Signal Process. 48(2), 151-157 (2001).

${ }^{44}$ F. V. Gasparyan, H. V. Asriyan, S. V. Melkonyan, and C. E. Korman, "Method of 1/f noise reduction and noise level manipulation in semiconductor based devices," U.S. patent application wo2011140541 (7 May 2010).

${ }^{45}$ J. Li, S. Pud, D. Mayer, and S. Vitusevich, "Advanced fabrication of $\mathrm{Si}$ nanowire FET structures by means of a parallel approach," Nanotechnology 25, 275302-1-7 (2014).

${ }^{46}$ D. E. Presnov, S. V. Amitanov, P. A. Krutitskii, V. V. Kolibasova, I. A. Devyatov, V. A. Krupenin, and I. I. Soloviev, "A highly pH-sensitive nanowire field-effect transistor based on silicon on insulator," Beilstein J. Nanotechnol. 4, 330-335 (2013).

${ }^{47}$ K. Bedner, V. A. Cuzenko, A. Tarasov, M. Wipf, R. L. Stoop, D. Just, S. Rigante, W. Fu, R. L. A. Minamisawa, Ch. David, M. Calame, J. Gobrecht, and Ch. Schönenberger, "pH response of silicon nanowire sensors: Impact of nanowire width and gate oxide," Sens. Mater. 25(8), 567-576 (2013) 